\title{
Synthesis and strong optical limiting response of graphite oxide covalently functionalized with gallium phthalocyanine
}

\author{
Yong-Xi Li ${ }^{1}$, Jinhui Zhu ${ }^{1}$, Yu Chen ${ }^{1}$, Jinjuan Zhang ${ }^{1}$, Jun Wang ${ }^{2,3}$, \\ Bin Zhang ${ }^{1}$, Ying $\mathrm{He}^{4}$ and Werner $\mathrm{J} \mathrm{Blau}^{3}$ \\ ${ }^{1}$ Key Lab for Advanced Materials, Institute of Applied Chemistry, East China University of \\ Science and Technology, 130 Meilong Road, Shanghai 200237, People's Republic of China \\ ${ }^{2}$ Key Lab of Materials for High-Power Laser, Shanghai Institute of Optics and Fine \\ Mechanics, Chinese Academy of Sciences, Shanghai 201800, People's Republic of China \\ ${ }^{3}$ Materials Ireland Polymer Research Centre, School of Physics and the Centre for Research \\ on Adaptive Nanostructures and Nanodevices (CRANN), Trinity College Dublin, \\ University of Dublin, Dublin 2, Ireland \\ ${ }^{4}$ Material Research and Analysis Center, School of Materials Science and Engineering, East \\ China University of Science and Technology, 130 Meilong Road, Shanghai 200237, \\ People's Republic of China
}

E-mail: chentangyu@yahoo.com and jwang@ siom.ac.cn

Received 16 November 2010, in final form 15 February 2011

Published 28 March 2011

Online at stacks.iop.org/Nano/22/205704

\begin{abstract}
A soluble graphite oxide (GO) axially substituted gallium phthalocyanine ( $\mathrm{PcGa}$ ) hybrid material (GO-PcGa) was for the first time synthesized by the reaction of $\mathrm{tBu}_{4} \mathrm{PcGaCl}$ with $\mathrm{GO}$ in anhydrous DMSO at $110^{\circ} \mathrm{C}$ in the presence of $\mathrm{K}_{2} \mathrm{CO}_{3}$. The formation of a $\mathrm{Ga}-\mathrm{O}$ bond between PcGa and GO has been confirmed by x-ray photoelectron spectroscopy. In contrast to GO, the D and G bands of GO-PcGa in the Raman spectrum are shifted to the lower wavenumbers by $\Delta v=11$ and $18 \mathrm{~cm}^{-1}$, respectively. At the same level of concentration of $0.1 \mathrm{~g} \mathrm{l}^{-1}$, GO-PcGa exhibit much larger nonlinear optical extinction coefficients and strong optical limiting performance than $\mathrm{GO}, \mathrm{tBu}_{4} \mathrm{PcGaCl}$ and $\mathrm{C}_{60}$ at both 532 and $1064 \mathrm{~nm}$, implying a remarkable accumulation effect as a result of the covalent link between GO and PcGa.

GO-PcGa possesses three main mechanisms for the nonlinear optical response-nonlinear light scattering, two-photon absorption and reverse saturable absorption for the $532 \mathrm{~nm}$ pulses and nonlinear light scattering for the $1064 \mathrm{~nm}$ pulses. $\mathrm{tBu}_{4} \mathrm{PcGaCl}$ does not make any significant contribution to the optical limiting at $1064 \mathrm{~nm}$, while GO-PcGa has a much greater optical limiting response than GO at this wavelength, this suggesting that the PcGa moiety could certainly play an unknown but important role in the GO-PcGa material system.
\end{abstract}

(Some figures in this article are in colour only in the electronic version)

\section{Introduction}

Phthalocyanines (Pcs) and their numerous analogs and derivatives are materials of tremendous importance in chemistry, materials science, physics, biology and medicine. A high architectural flexibility in a Pc structure facilitates the tailoring of their physical, optoelectronic and chemical parameters in a very broad range [1-6]. The exploitation of the chemical reactivity of the $\mathrm{Ga}-\mathrm{Cl}$ bond in the $\mathrm{R}_{x} \mathrm{PcGaCl}$ (R: peripheral substituents in the Pc macrocycle) can allow the preparation of a series of highly soluble axially substituted and bridged Pc complexes [7-14]. Axial substituents in 


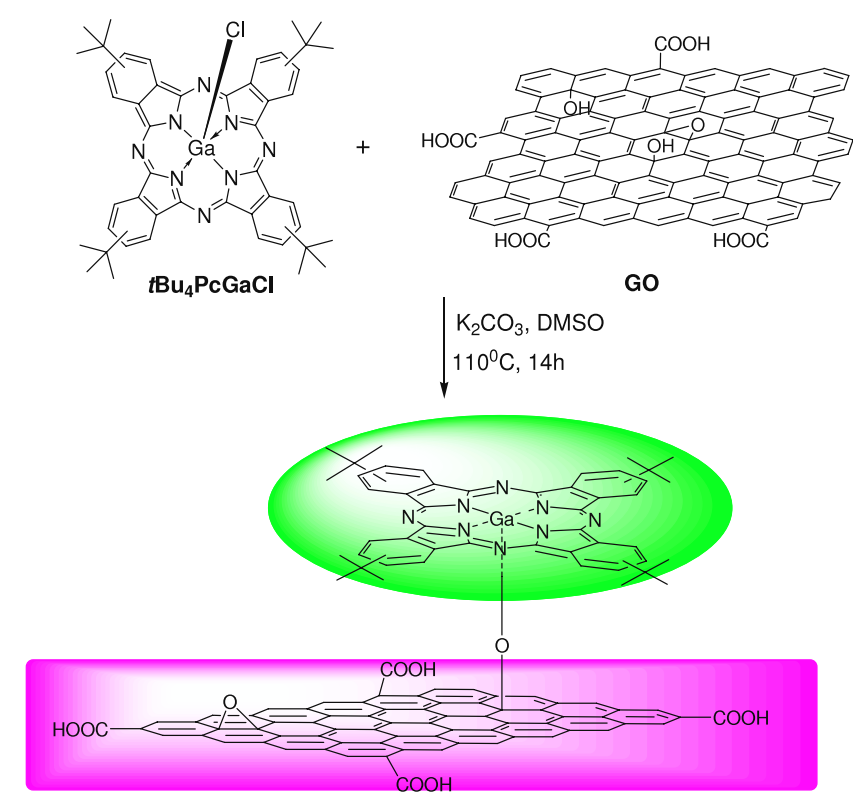

Scheme 1. Synthesis of GO-PcGa.

Pcs influence favorably nonlinear optical (NLO) absorption for the presence of a dipole moment perpendicular to the macrocycle in the axially substituted phthalocyanines [15-21]. Substitution and dimerization of the Pc monomer resulted in significant reductions in the saturation energy density of the material, displaying clear evidence of the usefulness of structurally modifying the phthalocyanine unit. Similar to indium phthalocyanines, gallium phthalocyanines are also one of the most promising materials that have been investigated as limiters of intense light.

Graphene discovered most recently has attracted considerable interest owing to their long-range $\pi$-conjugation, yielding extraordinary thermal, mechanical and electrical properties [22-29]. The ultrafast carrier dynamics and large absorption of incident light per layer make graphene a fast saturable absorber over a wide spectral range [30-32]. Wang et al [31] observed a significant NLO response of graphene dispersions to nanosecond laser pulses at 532 and $1064 \mathrm{~nm}$, implying a potential broadband optical limiting (OL) application. Nonlinear light scattering (NLS) arising from the formations of solvent bubbles and microplasmas is the principal mechanism for OL. We also observed the NLO and OL properties of graphene families, including graphite oxide (GO; the term 'GO' has been an acronym of graphite oxide for a long time. It should be noted that many papers are using 'GO' for graphene oxide which might be shortened in other ways.) nanosheets, graphene nanosheets (GNSs), GO nanoribbons (GONRs) and graphene nanoribbons (GNRs) at 532 and $1064 \mathrm{~nm}$ [32]. GNSs, GONRs and GNRs exhibited broadband NLO and OL properties. NLS and two-photon absorption (TPA) were found to have strong effects on the NLO and OL responses of the graphite nanostructures.

The chemistry of graphene reported in the literature mainly concerns the chemistry of GO [25, 33-35] because GO has chemically reactive oxygen functionality, including carboxylic acid groups and ketone groups at the edges of GO, and epoxy and hydroxyl groups on the basal planes. In our previous work, we synthesized $p$-chlorophenoxygallium(III)2,(3)-tetra(tert-butyl)phthalocyanine $\left[\mathrm{tBu}_{4} \mathrm{PcGa}(p\right.$-CPO)] by the reaction of $\mathrm{tBu}_{4} \mathrm{PcGaCl}$ with $p$-chlorophenol in anhydrous DMSO at $110^{\circ} \mathrm{C}$ in the presence of $\mathrm{K}_{2} \mathrm{CO}_{3}$ [13]. This idea persuaded us to utilize the reactive activity of hydroxyl groups on the basal planes of GO to prepare a first soluble GO axially functionalized gallium phthalocyanine (GO-PcGa) material, as shown in scheme 1. This material displayed excellent strong OL responses at 532 and $1064 \mathrm{~nm}$ due to the effective combination of the different NLO mechanisms.

\section{Experimental section}

\subsection{Materials and methods}

The operations for synthesis prior to the termination reaction were carried out under purified argon. All chemicals were purchased from Aldrich and used without further purification. Organic solvents were purified, dried and distilled under dry nitrogen. Purified natural graphite was purchased from Shanghai Yifan's Graphite Co. Ltd. $\mathrm{tBu}_{4} \mathrm{PcGaCl}$ was synthesized according to the literature reported by us [21].

Fourier transform infrared (FTIR) spectra were recorded on a Nicolet Nagma-IR 550 spectrophotometer using $\mathrm{KBr}$ pellets. The ultraviolet/visible (UV/vis) absorption spectral measurements were carried out with a Shimadzu UV-2450 spectrophotometer. Thermal properties of the samples were measured using a Perkin-Elmer Pyris 1 thermogravimetric analyzer in a flowing $\left(100 \mathrm{ml} \mathrm{min}^{-1}\right)$ nitrogen atmosphere. Steady-state fluorescence spectra were measured on a Horiba Jobin Yvon Fluoromax-4 spectrofluorometer. The sample for the fluorescence measurement was dissolved in dry tetrahydrofuran (THF), filtered, transferred to a long quartz cell, and then capped and bubbled with high pure argon (without $\mathrm{O}_{2}$ and moisture) for at least $15 \mathrm{~min}$ before measurement. X-ray photoelectron spectroscopy (XPS) measurements were carried on a Thermo ESCALAB 250 spectrometer with a monochromatized $\mathrm{Al} \mathrm{Kr}$ x-ray source (1486.6 eV photons) at a constant dwell time of $100 \mathrm{~ms}$ and a pass energy of $20 \mathrm{eV}$. Raman spectra were taken at room temperature with a MicroRaman System RM3000 spectrometer and an argon ion laser operating at a wavelength of $514.5 \mathrm{~nm}$ as the excitation source.

To measure the linear and NLO coefficients, GOPcGa was dispersed in $N, N^{\prime}$-dimethylformamide (DMF) at a concentration of $1.0 \mathrm{~g} \mathrm{l}^{-1}$, followed by $30 \mathrm{~min}$ ultrasonic processing. For the sake of fully evaluating the NLO performance of $\mathrm{GO}-\mathrm{PcGa}, \mathrm{GO}, \mathrm{tBu}_{4} \mathrm{PcGaCl}$ as well as $\mathrm{C}_{60}$ dispersions were prepared in the same way. All compounds show excellent dispersibility in DMF. As shown in figure 1, the GO dispersions exhibit a brownish color while the GO-PcGa dispersions possess a dark-green color, indicating the existence of the Pc moiety. The solubility of the GO-PcGa is mainly dependent on the percentage of PcGa grafted onto GO.

Linear extinction (absorption and/or scattering) coefficients were measured at a low level of incident light intensity. The linear extinction coefficient, $\alpha_{0}$, is defined by $T=$ 


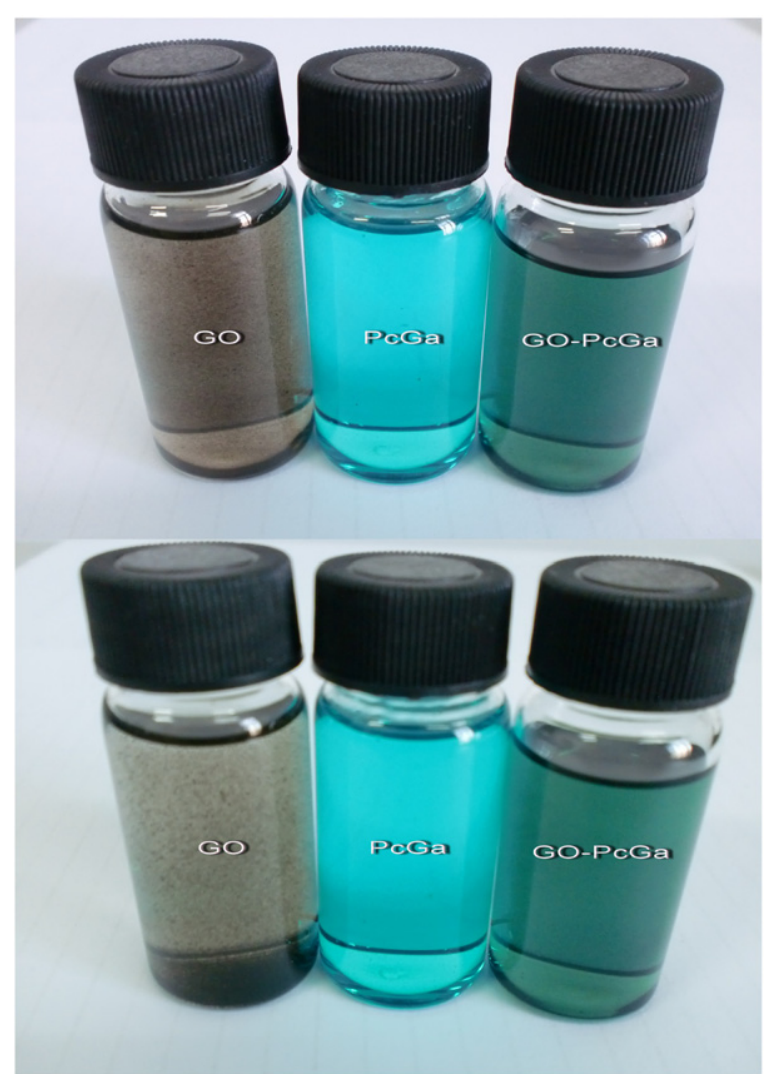

Figure 1. Digital pictures of the samples dispersed in chloroform: (above) dispersions immediately after sonication; and (bottom) dispersions $1 \mathrm{~h}$ after sonication. The concentration for each sample: $1 \mathrm{mg} \mathrm{ml}^{-1}$.

$\exp \left(\alpha_{0} L\right)$, where $T$ defines the ratio of transmitted to incident laser light and $L=0.1 \mathrm{~cm}$ is the sample thickness. The NLO properties of the GO-PcGa, GO, $\mathrm{PcGaCl}$ and $\mathrm{C}_{60}$ dispersions were studied using the open aperture $Z$-scan technique, which is widely adopted to investigate the nonlinear absorption, scattering and refraction processes. This measures the total transmittance through the material as a function of incident laser intensity, while the sample is gradually moved through the focus of a lens (along the $z$ axis). Effective extinction (absorption and/or scattering) coefficients can be deduced by the theory reported previously [20, 36]. The normalized transmittance as a function of position $z, T_{\text {Norm }}(z)$, is given by $T_{\text {Norm }}(z)=\log \left[1+q_{0}(z)\right] / q_{0}(z)$, where $q_{0}(z)=q_{00} /[1+$ $\left.\left(z / z_{0}\right)^{2}\right], z_{0}$ is the diffraction length of the beam. $q_{00}=$ $\beta_{\text {eff }} I_{0} L_{\text {eff }} . \quad \beta_{\text {eff }}$ is the effective intensity-dependent nonlinear extinction (NLE) coefficient and $I_{0}$ is the intensity of the light at focus. $L_{\text {eff }}$ is known as the effective length of the sample defined in terms of the linear absorption coefficient, $\alpha_{0}$, and the true optical path length through the sample, $L, L_{\text {eff }}=$ $\left[1-\exp \left(-\alpha_{0} L\right)\right] / \alpha_{0}$

In this work, the $Z$ scan was carried out by employing a $Q$-switched Nd:YAG laser of $6 \mathrm{~ns}$ pulses, operated at the fundamental $1064 \mathrm{~nm}$ and its second harmonic, $532 \mathrm{~nm}$, with a repetition rate of $10 \mathrm{~Hz}$. The laser beam was tightly focused with a $9 \mathrm{~cm}$ focus lens, after spatially removing higher-order modes. Meanwhile, another focusing lens was set up at $\sim 35^{\circ}$

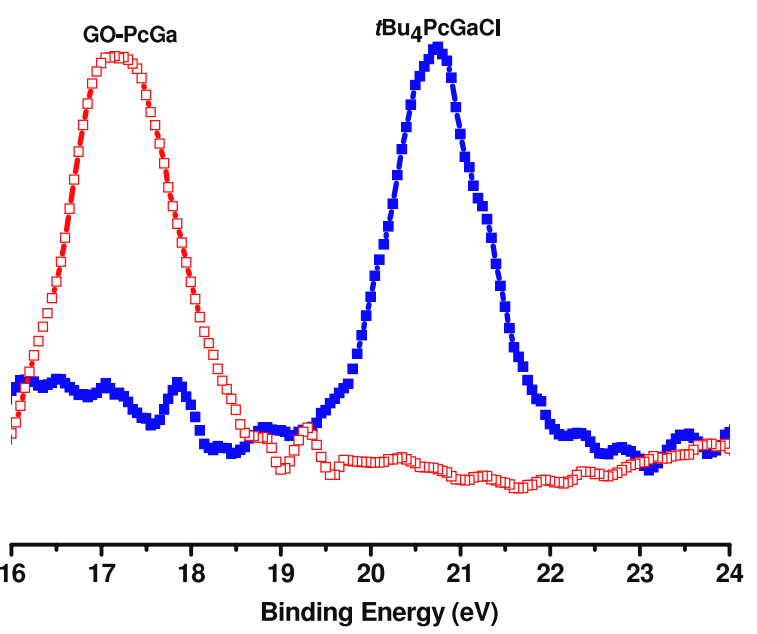

Figure 2. The Ga 3d XPS spectra of the samples.

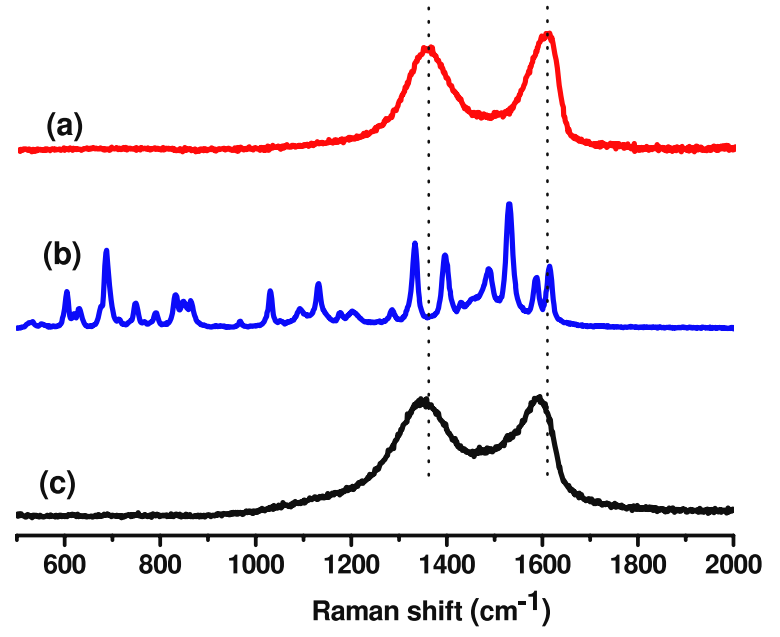

Figure 3. Raman spectra of (a) $\mathrm{GO}$, (b) $\mathrm{tBu}_{4} \mathrm{PcGaCl}$ and (c) GO-PcGa at $514 \mathrm{~nm}$.

to the direct incident beam to monitor the scattered light from the dispersions. All samples were examined in $0.1 \mathrm{~cm}$ quartz cells.

\subsection{Preparation of $G O$}

Twelve grams of graphite were suspended in $500 \mathrm{ml}$ of concentrated $\mathrm{H}_{2} \mathrm{SO}_{4}$ in a 11 round-bottomed flask under vigorous stirring. Six grams of $\mathrm{KMnO}_{4}$ were then added gradually with stirring and cooling so that the temperature was maintained below $10^{\circ} \mathrm{C}$. The stirring was then continued for $2 \mathrm{~h}$ at $35^{\circ} \mathrm{C}$, followed by the addition of $300 \mathrm{ml}$ of deionized water and stirring for another $15 \mathrm{~min}$. Finally the content of the flask was poured into 11 of deionized water and a sufficient amount of $\mathrm{H}_{2} \mathrm{O}_{2}(50 \mathrm{ml}$ of a $30 \%$ aqueous solution) was added to destroy the excess permanganate. Graphite oxide was isolated by centrifugation or filtration through a sintered glass filter, washed with dilute $\mathrm{HCl}$ until no sulfates were detected, and then dried for 10 days over $\mathrm{P}_{2} \mathrm{O}_{5}$ in a vacuum oven before use. $21 \mathrm{~g}$ of purified GO was obtained. 

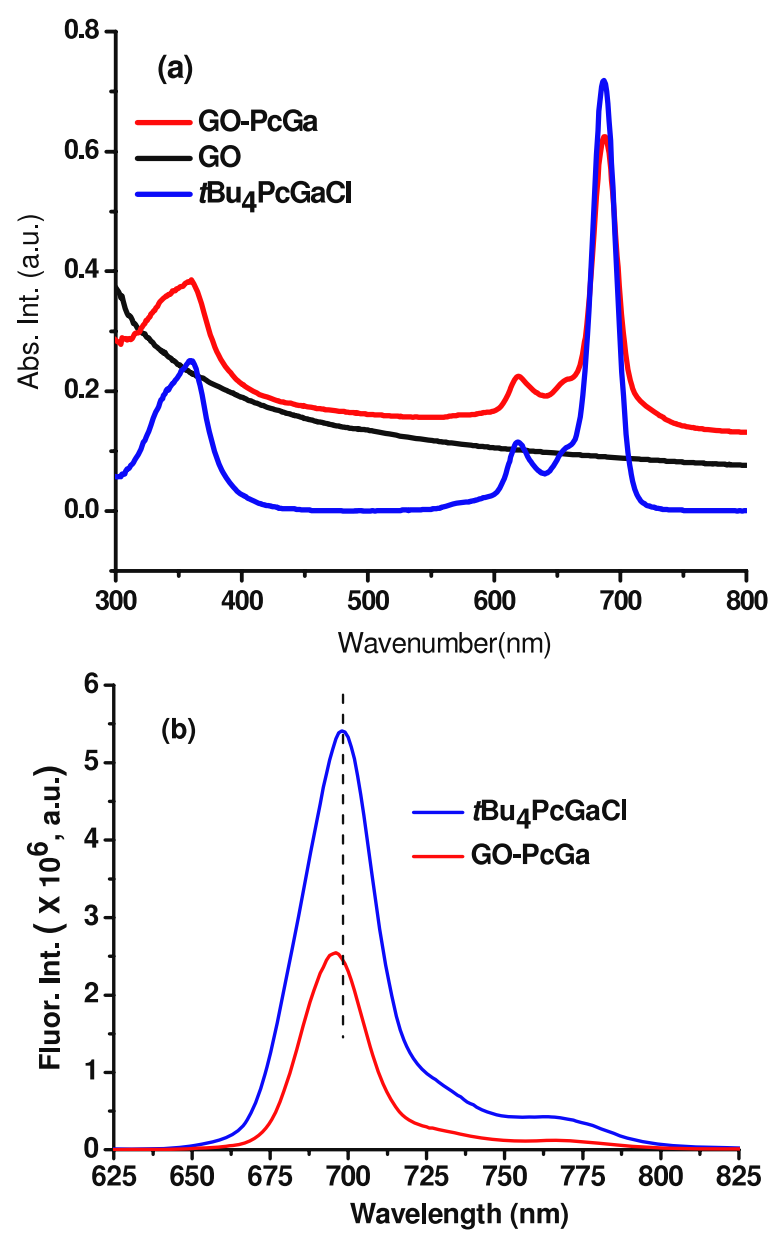

Figure 4. (a) UV/vis absorption and (b) photoluminescence $\left(\lambda_{\mathrm{ex}}=355 \mathrm{~nm}\right)$ spectra of the samples in DMF.

\subsection{Synthesis of $G O-P c G a$}

To a stirred solution of $\mathrm{GO}(50 \mathrm{mg})$ and $\mathrm{tBu}_{4} \mathrm{PcGaCl}^{3 \mathrm{~g}}$ (250 mg) in anhydrous dimethyl sulfoxide (DMSO, $60 \mathrm{ml}$ ) was slowly added $\mathrm{K}_{2} \mathrm{CO}_{3}(0.21 \mathrm{~g})$ under purified argon. After refluxing at $110^{\circ} \mathrm{C}$ for $14 \mathrm{~h}$, the temperature was allowed to cool to room temperature, while stirring was maintained for a total of $1 \mathrm{~h}$. The mixture was poured into ice water $(200 \mathrm{ml})$ and vacuum-filtered through a single layer nylon film $(\varphi 0.22 \mu \mathrm{m})$. The black solid on the nylon film was collected, washed with anhydrous $\mathrm{Et}_{2} \mathrm{O}$ until the filtrate became colorless and then dried at $60{ }^{\circ} \mathrm{C}$ in vacuo for $10 \mathrm{~h}$. Yield: $180 \mathrm{mg}$. UV/vis $\left(\mathrm{CHCl}_{3}\right): \lambda\left(\mathrm{nm}^{-1}\right)=688,657(\mathrm{sh}), 618,360 ; \mathrm{PL}$ $\left(\mathrm{DMF}, \lambda_{\mathrm{ex}}=355 \mathrm{~nm}\right): \lambda_{\max }\left(\mathrm{nm}^{-1}\right)=696$.

\section{Results and discussion}

The covalent attachment of the PcGa moieties onto the surface of GO was confirmed by XPS, as shown in figure 2. The $\mathrm{Ga} 3 \mathrm{~d}$ XPS spectrum of $\mathrm{tBu}_{4} \mathrm{PcGaCl}$ shows a peak at $20.8 \mathrm{eV}$ corresponding to the $\mathrm{Ga}$ in the $\mathrm{Ga}-\mathrm{Cl}$ bond. The introduction of GO into the axial position of PcGa resulted in significant blueshift of the peak of gallium functionality relative to $\mathrm{tBu}_{4} \mathrm{PcGaCl}$. The peak at $17.2 \mathrm{eV}$ is assigned to the $\mathrm{Ga}$

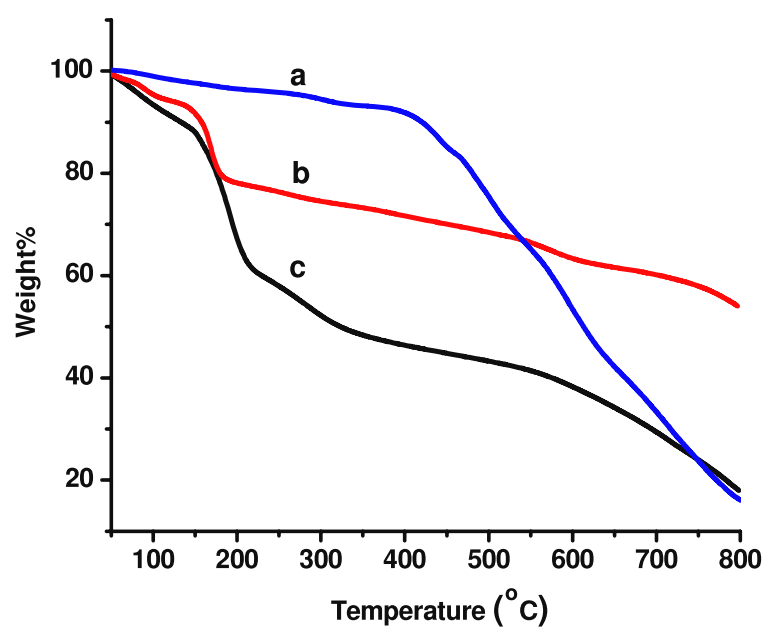

Figure 5. TGA curves of (a) $\mathrm{tBu}_{4} \mathrm{PcGaCl}$, (b) GO-PcGa and (c) GO. Heating rate: $10^{\circ} \mathrm{C} \mathrm{min}^{-1}$.

in the $\mathrm{Ga}-\mathrm{O}$ bond. The Raman spectrum of GO (figure 3, $\lambda_{\text {ex }}=514.5 \mathrm{~nm}$ ) shows two prominent bands at about 1355 (D band) and 1603 (G band) $\mathrm{cm}^{-1}$. In contrast to GO, the D and $\mathrm{G}$ bands of GO-PcGa are shifted to the lower wavenumbers by $\Delta v=11$ and $18 \mathrm{~cm}^{-1}$, respectively. The D- to G-band intensity ratios $\left(I_{\mathrm{D}} / I_{\mathrm{G}}\right)$ increased from 0.88 for Go to 1.01 for GO-PcGa. Usually functionalization of GO and RGO would lead to enhancement of the $I_{\mathrm{D}} / I_{\mathrm{G}}$ ratio because the $\mathrm{D}$ band has been used to monitor the process of covalent functionalization which transforms $\mathrm{sp}^{2}$ to $\mathrm{sp}^{3}$ sites, while the $\mathrm{G}$ band could be utilized to estimate the level and distribution of modification.

The electronic absorption spectra of Pcs are characterized by an intense $\mathrm{Q}$ band in the red end of the visible spectrum of light between 600 and $700 \mathrm{~nm}$, and a B band at 300$400 \mathrm{~nm}$ in the blue end of the visible spectrum. The influence of different axial substituents on the electronic structure of the phthalocyanine macrocycles is usually very small [7-21, 37-40]. As a result, GO-PcGa displays a linear UV/vis spectrum characterized by a weak redshift of the Q band relative to $\mathrm{tBu}_{4} \mathrm{PcGaCl}$ (figure 4). Upon excitation with a $355 \mathrm{~nm}$ laser, the photoluminescence spectrum of $\mathrm{tBu}_{4} \mathrm{PcGaCl}$ shows a red emission band at $\lambda=698 \mathrm{~nm}$, while the emission maximum of GO-PcGa is shifted to the blue by $\Delta \lambda=2 \mathrm{~nm}$, followed by a significant decrease of emission intensity when compared to that of $\mathrm{tBu}_{4} \mathrm{PcGaCl}$, suggesting that the quenching process is probably due to the electron-transfer process from PcGa to ${ }^{1} \mathrm{GO}^{*}$.

The thermal properties of GO [41] and GO-PcGa were evaluated by thermogravimetric analysis (TGA). From figure 5. it can be seen that GO is thermally unstable and suffers $15 \%$ weight loss upon heating to $100^{\circ} \mathrm{C}$. In agreement with previous reports in the literature for graphene, the main mass loss $(\sim 30 \%)$ takes place around $200^{\circ} \mathrm{C}$ and is ascribed to the decomposition of labile oxygen functional groups present in the material. $A \sim 15 \%$ weight loss of GO over the entire temperature range above $300{ }^{\circ} \mathrm{C}$ can be attributed to the removal of more stable oxygen functionalities. $\mathrm{tBu}_{4} \mathrm{PcGaCl}$ shows good thermal stability, with an onset decomposition temperature of $431^{\circ} \mathrm{C}$. After coupling of PcGa to GO, the 

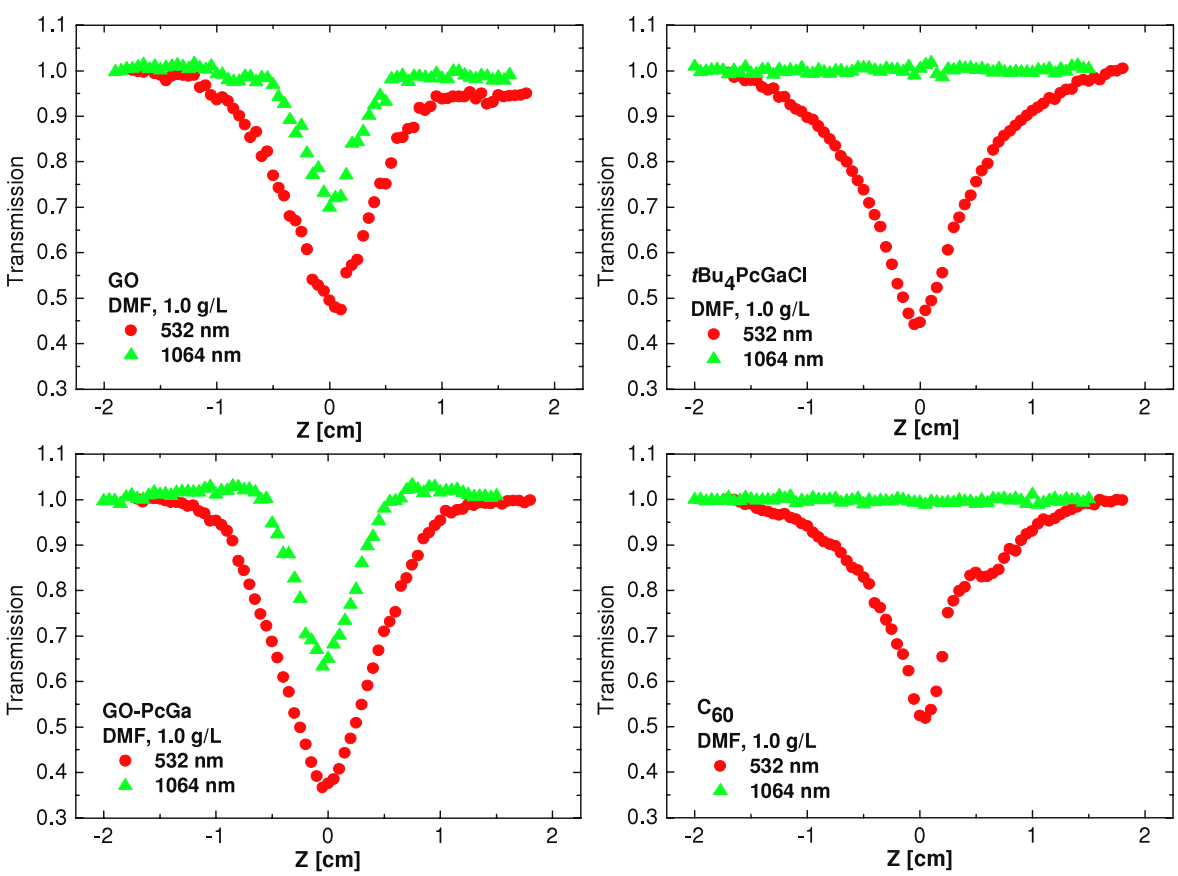

Figure 6. Open aperture $Z$ scans of the samples at 532 and $1064 \mathrm{~nm}$.

Table 1. Linear and NLO coefficients of the samples in DMF at a concentration of $1.0 \mathrm{~g}^{-1} \cdot \alpha_{0}$ : linear extinction coefficients; $\beta_{\text {eff }}$ : nonlinear extinction coefficient; $\operatorname{Im}\left\{\chi^{(3)}\right\}$ : the imaginary third-order susceptibility.

\begin{tabular}{|c|c|c|c|c|c|c|c|c|}
\hline \multirow[b]{2}{*}{ Name } & \multicolumn{4}{|c|}{$532 \mathrm{~nm}$} & \multicolumn{4}{|c|}{$1064 \mathrm{~nm}$} \\
\hline & $T(\%)$ & $\alpha_{0}\left(\mathrm{~cm}^{-1}\right)$ & $\beta_{\text {eff }}\left(\mathrm{cm} \mathrm{GW}^{-1}\right)$ & $\begin{array}{l}\operatorname{Im}\left\{\chi^{(3)}\right\} \\
\left(\times 10^{-12}, \text { esu }\right)\end{array}$ & $T(\%)$ & $\alpha_{0}\left(\mathrm{~cm}^{-1}\right)$ & $\beta_{\text {eff }}\left(\mathrm{cm} \mathrm{GW}^{-1}\right)$ & $\begin{array}{l}\operatorname{Im}\left\{\chi^{(3)}\right\} \\
\left(\times 10^{-12}, \text { esu }\right)\end{array}$ \\
\hline GO-PcGa & 34.3 & 10.70 & $67.33 \pm 0.63$ & $23.19 \pm 0.22$ & 39.7 & 9.24 & $11.82 \pm 0.38$ & $8.15 \pm 0.26$ \\
\hline GO & 51.9 & 6.55 & $28.10 \pm 1.41$ & $9.68 \pm 0.49$ & 59.9 & 5.13 & $6.08 \pm 0.78$ & $4.19 \pm 0.54$ \\
\hline $\mathrm{tBu}_{4} \mathrm{PcGaCl}$ & 87.6 & 1.33 & $28.4 \pm 0.16$ & $9.79 \pm 0.05$ & 93.0 & 0.72 & NA & NA \\
\hline $\mathrm{C}_{60}$ & 83.8 & 1.76 & $22.57 \pm 0.80$ & $8.55 \pm 0.30$ & 95.4 & 0.47 & NA & NA \\
\hline
\end{tabular}

resultant GO-PcGa complex is thermally more stable when compared with GO. When heated to $800^{\circ} \mathrm{C}$, GO-PcGa still retains about $54 \%$ of the original mass.

Figure 6 shows the typical open aperture $Z$-scan data for the GO-PcGa, GO, $\mathrm{tBu}_{4} \mathrm{PcGaCl}$ and $\mathrm{C}_{60}$. All $Z$ scans performed at $532 \mathrm{~nm}$ exhibited a reduction in the transmission on the focus of the lens, indicating a prominent OL effect. For $1064 \mathrm{~nm}$ irradiation both GO-PcGa and GO dispersions show clear NLO responses while $\mathrm{tBu}_{4} \mathrm{PcGaCl}$ and $\mathrm{C}_{60}$ solutions do not respond at this wavelength. As we have reported previously [31], pristine graphene dispersions exhibit a broadband NLO effect for nanosecond (ns) laser pulses at both 532 and $1064 \mathrm{~nm}$, resulting from the thermally induced NLS. In contrast, the NLO response of GO for ns pulses at $532 \mathrm{~nm}$ is attributed to a combination of TPA and NLS [32], in which the latter plays a major role since the TPA is much more pronounced for ps pulses than ns pulses [42, 43]. For the ns pulses at $1064 \mathrm{~nm}$, the NLS dominates the OL response of $\mathrm{GO}$, similar to that of the pristine graphene dispersions. Different from the zero-bandgap graphene, there is a finite bandgap in GO, depending on the functionalization by oxygen-containing groups [44]. Thus, we cannot rule out the possibility of nonlinear absorptions, i.e. multi-photonabsorption and/or excited state absorption of GO at $1064 \mathrm{~nm}$. However, it is expected that the contribution of the nonlinear absorptions to the OL should be minor in comparison with the NLS. $\mathrm{tBu}_{4} \mathrm{PcGaCl}$ and $\mathrm{C}_{60}$ solutions have large reverse saturable absorption (RSA) at $532 \mathrm{~nm}$ but being incapable of absorbing nonlinearly at $1064 \mathrm{~nm}$ [7]. From the above analysis, it is credible that GO-PcGa possesses three main mechanisms for the NLO response-NLS, TPA and RSA for the $532 \mathrm{~nm}$ pulses and NLS for the $1064 \mathrm{~nm}$ pulses. The $Z$ scans of $\mathrm{GO}-\mathrm{PcGa}$ have a much deeper reduction in transmission than those of GO at the same level of incident fluence, indicating a stronger NLO response. In addition to the contribution of the GO moiety, the PcGa manifests its contribution in the enhanced NLO response at $532 \mathrm{~nm}$ due to RSA. Table 1 summarizes the linear and NLO coefficients of GO-PcGa, GO, $\mathrm{tBu}_{4} \mathrm{PcGaCl}$ and $\mathrm{C}_{60}$, where the nonlinear extinction coefficients $\beta_{\text {eff }}$ were deduced from the $Z$-scan fitting. At the same level of concentration of $0.1 \mathrm{~g}^{-1}$, of all the four materials, GO-PcGa exhibits the largest linear absorption coefficient, nonlinear extinction coefficient and imaginary third-order susceptibility at both 532 and $1064 \mathrm{~nm}$, implying a remarkable accumulation effect as a result of the covalent link between GO and PcGa. 

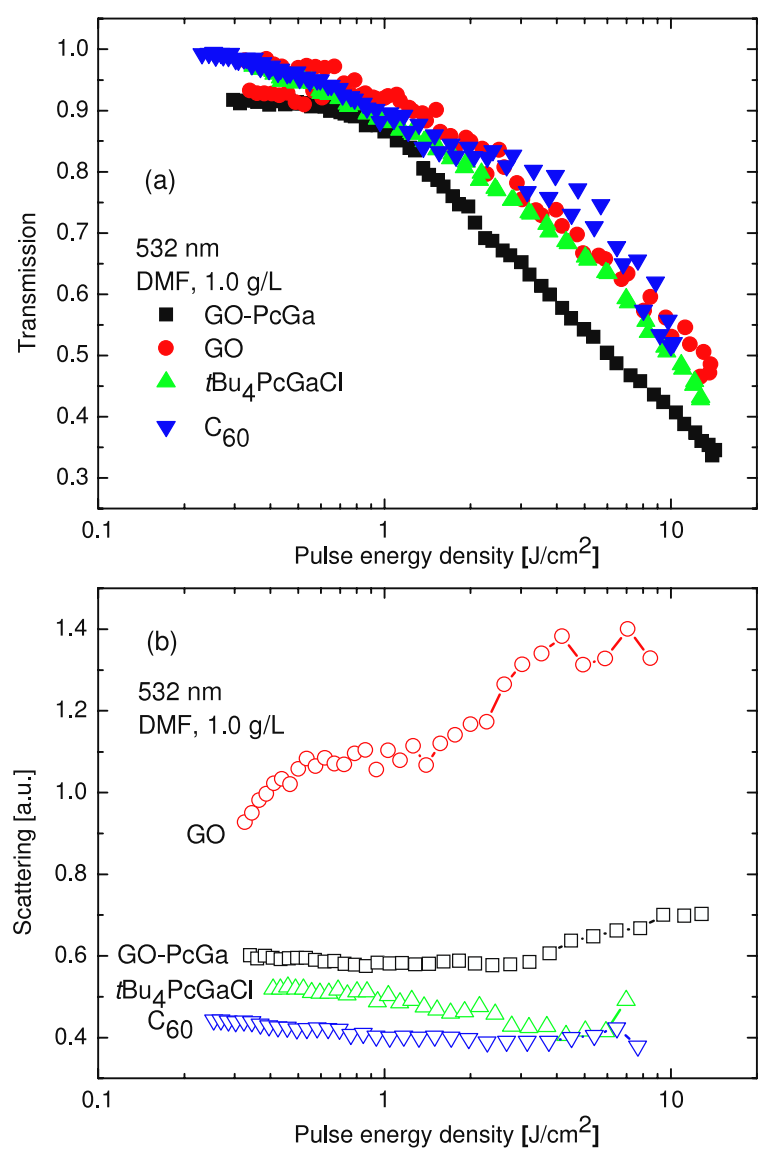

Figure 7. Nonlinear transmission (a) and scattering (b) of the samples at $532 \mathrm{~nm}$.

Figure 7 , in which the normalized transmission and the corresponding scattering were plotted as functions of input energy density $\left(\mathrm{J} \mathrm{cm}^{-2}\right)$, presents the OL performance of the samples at $532 \mathrm{~nm}$. At the same level of concentration, GOPcGa possesses much better OL performance than the other three materials. The enhanced OL response at $532 \mathrm{~nm}$ can be attributed to the effective combination of the different OL mechanisms, i.e. RSA in $\mathrm{tBu}_{4} \mathrm{PcGaCl}$ and NLS and TPA in GO. As shown in figure 7(b), significant light scattering signals were observed from GO-PcGa and GO. No scattering was seen from $\mathrm{tBu}_{4} \mathrm{PcGaCl}$ and $\mathrm{C}_{60}$, where only RSA dominates at $532 \mathrm{~nm}$.

Figure 8(a) shows the OL performance of the four materials at $1064 \mathrm{~nm}$. As expected, $\mathrm{tBu}_{4} \mathrm{PcGaCl}$ does not make any significant contribution to the OL at $1064 \mathrm{~nm}$, while GOPcGa has a much greater OL response than GO. On the other hand, it is seen in figure 8(b) that the scattered light from GO$\mathrm{PcGa}$ is much weaker than that from GO, which is explainable as the number density of the GO moiety in GO-PcGa should be smaller than that in the pristine GO dispersions. Therefore it is difficult to explain why GO-PcGa is superior to GO for OL if we consider the NLS as the only mechanism. The PcGa moiety could certainly play an unknown but important role in the GO-PcGa material system. Although the origin of such an improvement of the OL at $1064 \mathrm{~nm}$ is not clear yet, it is
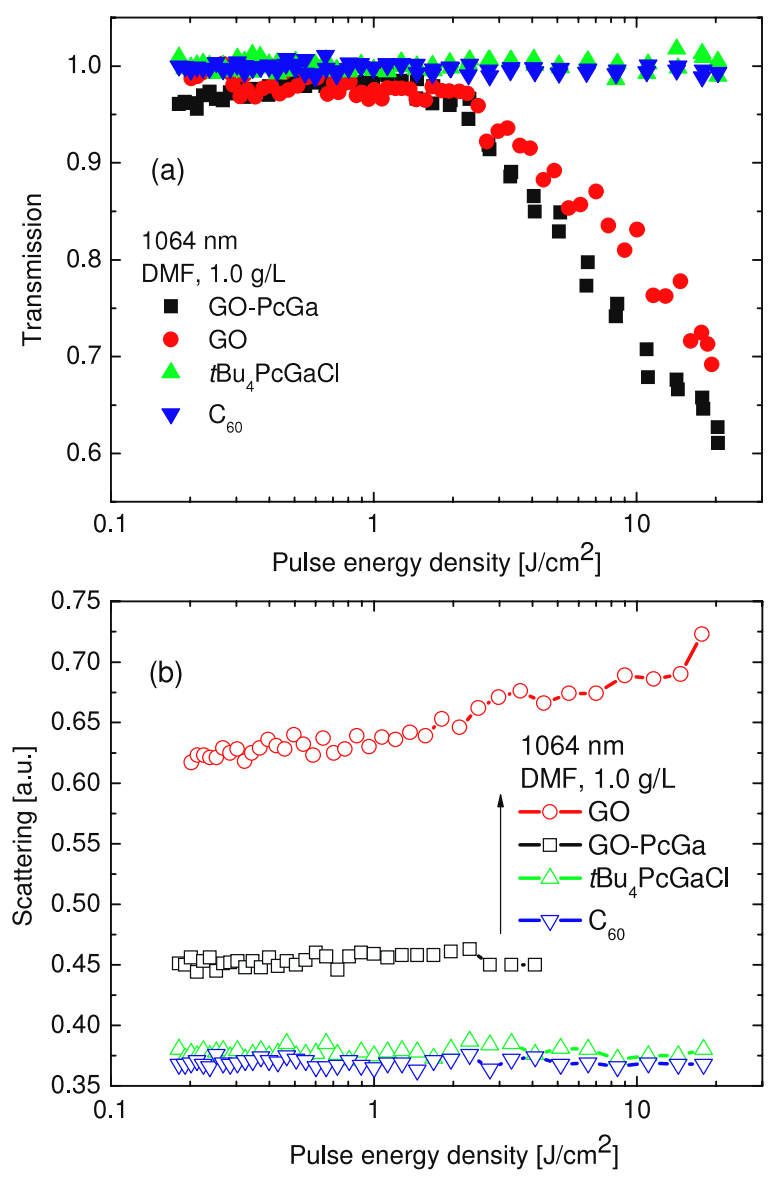

Figure 8. Nonlinear transmission (a) and scattering (b) of the samples at $1064 \mathrm{~nm}$.

undoubted that GO-PcGa has much better broadband NLO and OL performance than $\mathrm{GO}$ or $\mathrm{tBu}_{4} \mathrm{PcGaCl}$ alone.

In summary, a soluble graphite oxide axially substituted gallium phthalocyanine hybrid material was synthesized. At the same level of concentration, GO-PcGa exhibits much larger NLO extinction coefficients and strong OL performance than $\mathrm{GO}, \mathrm{tBu}_{4} \mathrm{PcGaCl}$ and $\mathrm{C}_{60}$ at both 532 and $1064 \mathrm{~nm}$, implying a remarkable accumulation effect as a result of the covalent link between GO and PcGa.

\section{Acknowledgments}

The authors are grateful for the financial support of the National Natural Science Foundation of China (20676034 and 20876046), the Ministry of Education of China (309013), the Fundamental Research Funds for the Central Universities, the Shanghai Municipal Educational Commission for the Shuguang Fellowship (08GG10) and the Shanghai Eastern Scholarship.

\section{References}

[1] Nalwa H S and Shirk J S in 1996 Phthalocyanines: Properties and Applications vol 4, ed C C Leznoff and A B P Lever (New York: VCH) p 83

[2] Hanack M and Lang M 1994 Adv. Mater. 6 819-33 
[3] Mckeown N B 1998 Phthalocyanine Materials: Synthesis, Structure and Function ed B Dunn, J W Goodby and A R West (Cambridge: Cambridge University Press)

[4] De La Torre G, Vazquez P, Agullo-Lopez F and Torres T 1998 J. Mater. Chem. 8 1671-83

[5] De La Torre G, Vazquez P, Agullo-Lopez F and Torres T 2004 Chem. Rev. 104 3723-50

[6] Nemykin V N and Lukyanets E A 2010 ARKIVOC 136-208

[7] Chen Y, Hanack M, Araki Y and Ito O 2005 Chem. Soc. Rev. 34 517-29

[8] Chen Y, Fujitsuka M, O'Flaherty S M, Hanack M, Ito O and Blau W J 2003 Adv. Mater. 15 899-902

[9] Chen Y, O'Flaherty S, Fujitsuka M, Hanack M, Subramanian L R, Ito O and Blau W J 2002 Chem. Mater. 14 5163-8

[10] Chen Y, Subramanian L R, Fujitsuka M, Ito O, O'Flaherty S M, Blau W J, Schneider T, Dini D and Hanack M 2002 Chem. Eur. J. 8 4248-54

[11] Claessens C G, Gouloumis A, Barthel M, Chen Y, Martin G, Agullo-Lopez F, Ledoux-Rak I, Zyss J, Hanack M and Torres T 2003 J. Porphyr. Phthalocya. 7 291-5

[12] Yang G Y, Hanack M, Lee Y W, Chen Y, Yuen M L K, Vagin S and Dini D 2003 Chem. Eur. J. 9 2758-62

[13] Chen Y, O'Flaherty S M, Hanack M and Blau W J 2003 J. Mater. Chem. 13 2405-8

[14] Bertagnolli H, Blau W J, Chen Y, Dini D, Feth M P, O'Flaherty S M, Hanack M and Krishnan V 2005 J. Mater. Chem. 15 683-9

[15] Chen Y, Hanack M, Blau W J, Dini D, Doyle J, Liu Y, Lin Y and Bai J 2006 J. Mater. Sci. 41 2169-85

[16] Chen Y, EI-Khouly M E, Sasaki M, Araki Y and Ito O 2005 Org. Lett. 7 1613-6

[17] Chen Y, EI-Khouly M E, Doyle J J, Lin Y, Liu Y, Notaras E, Blau W J and O'Flaherty S M 2008 Handbook of Organic Electronics and Photonics vol 2 (Stevenson Ranch, CA: American Scientific Publishers) pp 151-81

[18] Chen Y, Barthel M, Seiler M, Subramanian L R, Bertagnolli H and Hanack M 2002 Angew Chem. Int. Eng. Edn 41 3239-42

[19] Chen Y, Dini D, Hanack M, Fujitsuka M and Ito O 2004 Chem. Commun. 340-1

[20] O'Flaherty S M, Hold S V, Cook M J, Torres T, Chen Y, Hanack M and Blau W J 2003 Adv. Mater. 15 19-32

[21] Chen Y, Subramanian L R, Barthel M and Hanack M 2002 Eur. J. Inorg. Chem. 1032-4

[22] Wu J, Pisula W and Muellen K 2007 Chem. Rev. 107 718-47

[23] Chen F and Tao N J 2009 Acc. Chem. Res. 42 429-38
[24] Dreyer D R, Park S, Bielawski C W and Ruoff R S 2010 Chem. Soc. Rev. $39228-40$

[25] Park S and Ruoff R S 2009 Nat. Nanotechnol. 4 217-24

[26] Rao C N R, Sood A K, Subrahmanyam K S and Govindaraj A 2009 Angew. Chem. Int. Edn 48 7752-77

[27] Lee C, Wei X, Kysar J W and Hone J 2008 Science 321 385-8

[28] Stoller M D, Park S, Zhu Y, An J and Ruoff R S 2008 Nano Lett. 8 3498-502

[29] Zhang Y, Tan Y W, Stormer H L and Kim P 2005 Nature 438 201-4

[30] Sun Z, Hasan T, Torrisi F, Popa D, Privitera G, Wang F, Bonaccorso F, Basko D M and Ferrari A C 2010 ACS Nano 4 803-10

[31] Wang J, Hernandez Y, Lotya M, Coleman J N and Blau W J 2009 Adv. Mater. 21 2430-5

[32] Feng M, Zhan H and Chen Y 2010 Appl. Phys. Lett. 96033107

[33] Loh K P, Bao Q, Ang P K and Yang J 2010 J. Mater. Chem. $202277-89$

[34] Zhuang X D, Chen Y, Liu G, Li P P, Zhu C X, Kang E T, Noeh K G, Zhang B, Zhu J H and Li Y X 2010 Adv. Mater. 22 1731-5

[35] Zhang B, Chen Y, Zhuang X D, Liu G, Yu B, Kang E T and Li Y X 2010 J. Polym. Sci. A 48 2642-9

[36] Sheikbahae M, Said A A, Wei T H, Hagan D J and Vanstryland E W 1990 IEEE J. Quantum Electron. 26 760-9

[37] Hanack M, Schneider T, Barthel M, Shirk J S, Flom S R and Pong R G S 2001 Coord. Chem. Rev. 219-221 235-58

[38] Shirk J S, Pong R G S, Flom S R, Heckmann H and Hanack M 2000 J. Phys. Chem. A 104 1438-49

[39] Perry J W et al 1996 Science 273 1533-6

[40] Barthel M, Vagin S, Dini D and Hanack M 2002 Eur. J. Org. Chem. 3756-62

[41] Georgakilas V, Bourlinos A B, Zboril R, Steriotis T A, Dallas P, Stuboscd A K and Trapalisa C 2010 Chem. Commun. 46 1766-8

Becerril H A, Mao J, Liu Z, Stoltenberg R M, Bao Z and Chen Y 2008 ACS Nano 2 463-70

Paredes J I, Villar-Rodil S, Martínez-Alonso A and Tascón J M D 2008 Langmuir 24 10560-4

Li P P, Chen Y, Zhu J, Feng M, Zhuang X, Lin Y and Zhan H 2011 Chem. Eur. J. 17 780-5

[42] Wang J and Blau W J 2009 J. Opt. A: Pure Appl. Opt. 11024001

[43] Liu Z B, Wang Y, Zhang X L, Xu Y F, Chen Y S and Tian J G 2009 Appl. Phys. Lett. 9402192

[44] Boukhvalov D W and Katsnelson M I 2008 J. Am. Chem. Soc. 130 10697-701 